\title{
Distinguishing Direct from Indirect Interactions in Oscillatory Networks with Multiple Time Scales
}

\author{
Jakob Nawrath, ${ }^{1,2, *}$ M. Carmen Romano, ${ }^{3,4}$ Marco Thiel, ${ }^{3}$ István Z. Kiss, ${ }^{5}$ Mahesh Wickramasinghe, ${ }^{5}$ Jens Timmer, ${ }^{1,2,6}$ \\ Jürgen Kurths, ${ }^{3,7,8}$ and Björn Schelter ${ }^{1,2}$ \\ ${ }^{1}$ Physics Department, University of Freiburg, Hermann-Herder Str. 3, 79104 Freiburg, Germany \\ ${ }^{2}$ Center for Data Analysis and Modeling, University of Freiburg, Eckertstr. 1, 79104 Freiburg, Germany \\ ${ }^{3}$ Institute for Complex Systems and Mathematical Biology, University of Aberdeen, Aberdeen AB24 3UE, United Kingdom \\ ${ }^{4}$ Institute of Medical Sciences, Foresterhill, University of Aberdeen, Aberdeen AB25 2ZD, United Kingdom \\ ${ }^{5}$ Department of Chemistry, Saint Louis University, St. Louis, Missouri 63103, USA \\ ${ }^{6}$ Freiburg Institute for Advanced Studies, Albertstr. 19, 79104 Freiburg, Germany \\ ${ }^{7}$ Institute of Physics, Humboldt University Berlin, 12489 Berlin, Germany \\ ${ }^{8}$ Potsdam Institute for Climate Impact Research, 14412 Potsdam, Germany
}

(Received 5 August 2009; published 21 January 2010)

\begin{abstract}
We propose a method to infer the coupling structure in networks of nonlinear oscillatory systems with multiple time scales. The method of partial phase synchronization allows us to infer the coupling structure for coupled nonlinear oscillators with one well-defined time scale. The case of oscillators with multiple time scales has remained a challenge until now. Here, we introduce partial recurrence based synchronization analysis to tackle this challenge. We successfully apply the proposed method to model systems and experimental data from coupled electrochemical oscillators. The statistical significance of the results is evaluated based on a surrogate hypothesis test.
\end{abstract}

DOI: 10.1103/PhysRevLett.104.038701

PACS numbers: 05.45.Xt, 02.50.Sk, 89.20.-a

Oscillators in complex real-world systems typically exhibit multiple time scales. Examples are climate oscillations from El Niño or Southern Oscillation and the Monsoon, active drilling vibrations in engineering, stock market oscillations, and biological oscillators, such as neuronal oscillators with their spiking and bursting behavior and brain activity measured from electroencephalograms or functional magnetic resonance imaging. Here we focus on small networks consisting of a few nodes, which are typical for many applications, such as the detection of motifs embedded in larger networks or data measured from a few regions of interest in the brain [1]. One crucial step towards understanding the characteristic behavior of such systems consists in inferring the connection topology of their constituting parts based on synchronization properties. When studying the connectivity structure in networks of such systems, one faces two main challenges: (i) bivariate measures to quantify the degree of synchronization inevitably lead to false positive conclusions about the network structure due to confounding factors; (ii) due to multiple time scales in real-world systems, conventional techniques to quantify the degree of synchronization are not applicable. To approach the first challenge, partial spectral coherence was introduced for linear systems [2] and extended to nonlinear systems by partial phase synchronization [3]. These methods can distinguish direct from indirect interactions. However, investigations using partial phase synchronization are constrained by the requirement that the oscillatory systems under study are phase coherent; i.e., they have one well defined time scale. To tackle the second challenge, a synchronization measure based on the concept of recurrence in phase space was introduced [4,5] to detect and quantify synchronization in real-world systems with multiple time scales. This recurrence based method is bivariate, however, so that it cannot detect unequivocally the coupling configuration in a system consisting of three or more subsystems. Therefore, the challenge of distinguishing direct from indirect connections in oscillatory systems with multiple time scales remained unsolved. In this Letter, we generalize partial phase synchronization by introducing partial recurrence based synchronization (PARS) analysis. We demonstrate how this technique can be successfully applied to infer the connection topology of networks of oscillators with multiple time scales.

The method of partial phase synchronization was introduced [3] to distinguish direct from indirect interactions in networks of nonlinear oscillators with one predominant time scale. Consider, e.g., the network of coupled oscillators in Fig. 1(a). A bivariate measure for synchronization
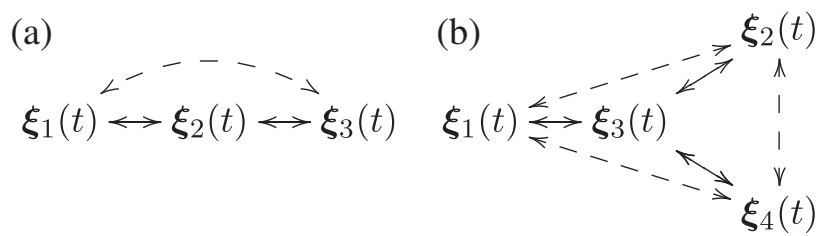

FIG. 1. Networks of coupled oscillators. Dashed arrows indicate indirect interactions. 
would detect synchronization between oscillator $\boldsymbol{\xi}_{1}(t)$ and $\boldsymbol{\xi}_{3}(t)$, in spite of them being coupled only via the mediating influence of $\boldsymbol{\xi}_{2}(t)$. Assuming that we have $N$ interdependent oscillatory systems $\boldsymbol{\xi}_{1}(t), \ldots, \boldsymbol{\xi}_{N}(t)$, where $\boldsymbol{\xi}_{i}(t) \in \mathbb{R}^{d}$ denotes the trajectory of oscillator $i$ in $d$-dimensional phase space and $t=1, \ldots, T$, the method of partial phase synchronization calculates first the synchronization indices

$$
\left|R_{k, l}\right|=\left|T^{-1} \sum_{t=1}^{T} \exp \left(i \Phi_{k, l}(t)\right)\right|, \quad\left|R_{k, l}\right| \in[0,1]
$$

for all pairs of oscillators $\boldsymbol{\xi}_{k}(t)$ and $\boldsymbol{\xi}_{l}(t)$. Here $\Phi_{k, l}(t)=$ $\left|\Phi_{k}(t)-\Phi_{l}(t)\right|$ is the phase difference of oscillators $\boldsymbol{\xi}_{k}(t)$ and $\boldsymbol{\xi}_{l}(t)$ at time $t$ [6,7]. The phase $\Phi(t)$ of an oscillator $\boldsymbol{\xi}(t)$ can be obtained using, e.g., the analytical signal approach $\tilde{x}(t)=x(t)+i x_{H}(t)=A(t) \exp i \Phi(t)$ [8] where $x(t)$ is an observed scalar time series from oscillator $\boldsymbol{\xi}(t)$, $x_{H}(t)$ is the Hilbert transform of $x(t)$, and $A(t)$ is the instantaneous amplitude. The analysis of the bivariate synchronization indices $R_{k, l}$ results in a Hermitian bivariate synchronization matrix

$$
\mathbf{R}=\left(\begin{array}{cccc}
1 & R_{1,2} & \ldots & R_{1, N} \\
R_{1,2}^{*} & 1 & \ldots & R_{2, N} \\
\vdots & \vdots & \ddots & \vdots \\
R_{1, N}^{*} & R_{2, N}^{*} & \ldots & 1
\end{array}\right) .
$$

Based on matrix inversion of the synchronization matrix $\mathbf{R}$, the partial phase synchronization index

$$
R_{k, l \mid z}=\left|R_{k, l}^{-1}\right| / \sqrt{R_{k, k}^{-1} R_{l, l}^{-1}}
$$

yields the mean phase coherence between $\boldsymbol{\xi}_{k}(t)$ and $\boldsymbol{\xi}_{l}(t)$ conditioned on the remaining processes $\left\{\boldsymbol{\xi}_{z}(t) \mid z=\right.$ $1, \ldots, N, z \neq k, l\}$ [3]. Thus, if the bivariate synchronization index $R_{k, l}$ is considerably different from zero, while the multivariate index $R_{k, l \mid z}$ vanishes, there is strong evidence for an indirect coupling between oscillators $\boldsymbol{\xi}_{k}(t)$ and $\boldsymbol{\xi}_{l}(t)$.

However, if the oscillators exhibit multiple time scales, the definition of the phase is not straightforward anymore, and the synchronization index defined in Eq. (1) cannot be calculated. This is because for an oscillator with a broadband spectrum there is no clear center of rotation if its trajectory is projected on a plane [8]. In these cases, we can detect phase synchronization by a measure that is based on the recurrences of a trajectory and considers phase synchronization in a more statistical sense [5]. The recurrences in phase space of an oscillator $\boldsymbol{\xi}(t)$ are represented by the recurrence matrix [9]

$$
P(\varepsilon)_{t, r}=\Theta(\varepsilon-\|\boldsymbol{\xi}(t)-\boldsymbol{\xi}(r)\|), \quad t, r=1, \ldots, T,
$$

where $\Theta(\cdot)$ is the Heaviside function, $\|\cdots\|$ is an appropriate norm, and $\varepsilon$ is a predefined threshold. An estimate of the probability $p(\varepsilon, \tau)$ that a system recurs to the $\varepsilon$ neigh- borhood of a former point of the trajectory after $\tau$ time steps is given by the diagonal-wise calculated $\tau$-recurrence rate $r_{\tau}(\varepsilon)$,

$$
\hat{p}(\varepsilon, \tau)=r_{\tau}(\varepsilon)=\frac{1}{T-\tau} \sum_{t=1}^{T-\tau} P(\varepsilon)_{t, t+\tau} .
$$

This measure can be considered as a generalized autocorrelation function [5], since it describes higher-order correlations among the points of the trajectory depending on $\tau$. Note that it is defined for a trajectory, and not restricted to scalar time series. For oscillatory systems, the function $p(\varepsilon, \tau)$ shows maxima at multiples of the main time scales of the system. If two oscillators $\boldsymbol{\xi}_{k}(t)$ and $\boldsymbol{\xi}_{l}(t)$ synchronize, their respective time scales adapt to each other, and hence, the positions of the maxima of their corresponding generalized autocorrelation functions $p_{k}(\varepsilon, \tau)$ and $p_{l}(\varepsilon, \tau)$ coincide. To quantify these coincidences, the cross correlation coefficient of the probabilities of recurrence

$$
\rho_{k, l}=\left\langle\hat{p}_{k}(\varepsilon, \tau) \hat{p}_{l}(\varepsilon, \tau)\right\rangle, \quad \rho_{k, l} \in[0,1]
$$

can be calculated. In the case of locked phase dynamics, the probability of recurrence is simultaneously maximal for both systems and hence $\rho_{k, l}$ significantly differs from zero. The applicability of this synchronization measure was demonstrated for a general class of non-phasecoherent and nonstationary systems and even for time series corrupted by strong noise [5].

To treat the multivariate case of $N$ interdependent oscillators based on the recurrence method, the matrix of the bivariate synchronization indices [Eq. (6)] $\boldsymbol{\rho}=$ $\left(\rho_{k, l}\right)_{k, l=1, \ldots, N}$ is introduced. This matrix has the same symmetry properties as the synchronization matrix in Eq. (2). Using the inverse matrix $\boldsymbol{\rho}^{-1}$, the partial recurrence based synchronization (PARS) index is defined as follows

$$
\rho_{k, l \mid z}=\left|\rho_{k, l}^{-1}\right| / \sqrt{\rho_{k, k}^{-1} \rho_{l, l}^{-1}},
$$

where $\rho_{k, l}^{-1}$ indicates the $(k, l)$ element of the inverse matrix $\boldsymbol{\rho}^{-1}$. This measure quantifies phase synchronization between two oscillators $\boldsymbol{\xi}_{k}(t)$ and $\boldsymbol{\xi}_{l}(t)$, conditioned on the remaining oscillators $\left\{\boldsymbol{\xi}_{z}(t) \mid z=1, \ldots, N, z \neq k, l\right\}$. Therefore, this method solves both crucial challenges in the analysis of networks of oscillators with multiple time scales: it distinguishes direct from indirect connections and it is applicable to real-world oscillators with a broadband power spectrum.

To illustrate the performance of PARS analysis, a network consisting of three funnel Rössler oscillators [10]

$$
\dot{\boldsymbol{\xi}}_{k}=\left(\begin{array}{c}
\dot{x}_{k} \\
\dot{y}_{k} \\
\dot{z}_{k}
\end{array}\right)=\left(\begin{array}{c}
-\omega_{k} y_{k}-z_{k}+\sum_{l \neq k} \mu_{k, l}\left(x_{l}-x_{k}\right) \\
\omega_{k} x_{k}+a y_{k} \\
0.1-\left(x_{k}-8.5\right) z_{k}
\end{array}\right) \text {, }
$$

for $k=1,2,3$, is examined. The subsystems are non- 
identical $\left(\omega_{1}=0.98, \omega_{2}=1.02\right.$, and $\left.\omega_{3}=1.06\right)$ and diffusively coupled via their $x$ components. The oscillator $\boldsymbol{\xi}_{2}(t)$ is bidirectionally coupled to both $\boldsymbol{\xi}_{1}(t)$ and $\boldsymbol{\xi}_{3}(t)$, whereas there is no direct coupling between $\boldsymbol{\xi}_{1}(t)$ and $\boldsymbol{\xi}_{3}(t)$ [Fig. 1(a)]. The parameter $a=0.2925$ is chosen such that the oscillators are in the highly non-phase-coherent funnel regime [10]; i.e., they exhibit multiple time scales. The set of differential equations was numerically integrated and sampled at $20 \mathrm{~Hz}$, starting from randomly chosen initial conditions. The first 10000 transient data points were discarded and time series consisting of 24850 data points were analyzed. From the $x$ component, the phase space was reconstructed by delay embedding with delay time 30 and embedding dimension 6 . The embedding parameters were estimated by the mutual information and the method of false nearest neighbors [11]. For the computation of the recurrence matrices, the threshold $\varepsilon$ was chosen so that a recurrence rate of 0.1 resulted [cf. Eq. (5)] [9].

In Fig. 2 the bivariate indices $\rho_{k, l}$ and the multivariate PARS indices $\rho_{k, l \mid z}$ are compared for $\mu=\mu_{1,2}=\mu_{2,3}$ varied in the range $[0,0.3]$. To evaluate the statistical significance of the results, the method of twin surrogates is utilized [12]. A twin surrogate of a given trajectory corresponds to an independent trajectory of the same underlying system starting at different initial conditions. The significance level for $\rho_{k, l}$ was obtained from the synchronization indices $\rho_{k, l_{\text {sur }}}$, where the oscillator $\boldsymbol{\xi}_{l}(t)$ was replaced by its twin surrogate. Alternatively, one could have replaced oscillator $\boldsymbol{\xi}_{k}(t)$ by its surrogate. The $1 \%$ significance level was given by the second highest value from 100 realizations of the surrogates. To compute the significance level of the PARS indices, these values were arranged in the matrix $\boldsymbol{\rho}_{\text {surr }}$, which was then inverted and renormalized following Eq. (7). The bivariate measures indicate that each oscillator is connected to all others, since for sufficiently strong coupling strength $\mu$ the indices $\rho_{k, l}$ are significant for all $\{k, l\}$ combinations [Figs. 2(a)-2(d)].

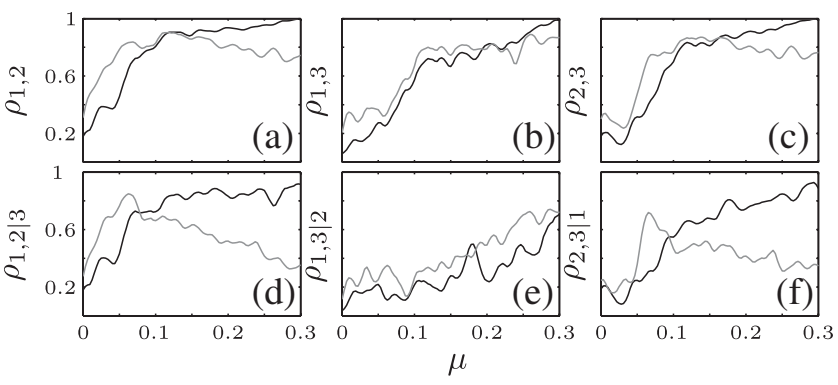

FIG. 2. (a)-(c) Bivariate synchronization indices and (d)(f) multivariate PARS indices depending on the coupling strength $\mu$ for three interdependent funnel Rössler systems exhibiting multiple time scales. The significance levels (gray) are based on the method of twin surrogates. The curves have been smoothed over 8 data points. (e) Only the PARS indices correctly determine the indirect coupling between oscillators $\boldsymbol{\xi}_{1}(t)$ and $\boldsymbol{\xi}_{3}(t)$.
But computing the PARS indices, we see that only $\rho_{1,2 \mid 3}$ and $\rho_{2,3 \mid 1}$ are significant for large $\mu$, correctly detecting that the connection between oscillators $\boldsymbol{\xi}_{1}(t)$ and $\boldsymbol{\xi}_{3}(t)$ is indirect [Figs. 2(d)-2(f)]. We emphasize that the same results are obtained when using "natural" surrogates [12], i.e., using the differential equations of the Rössler oscillators to generate independent trajectories.

In Fig. 3 we investigate a network consisting of four coupled funnel oscillators $\left(\omega_{1}=0.98, \omega_{2}=1.04, \omega_{3}=1\right.$ and $\left.\omega_{4}=0.94\right)$. All oscillators are coupled to oscillator $\boldsymbol{\xi}_{3}(t)$, without any further direct connections [Fig. 1(b)]. Furthermore, we add 50\% observational noise containing auto and cross correlations to all four oscillators. This coupling configuration is correctly detected by the PARS indices [Fig. 3]. Also for other levels of observational noise between 5\% and 50\% the PARS analysis is able to reveal the actual coupling structure (results not shown). We emphasize that other multivariate techniques, including the linear ones, usually lead to false positive conclusions about the coupling configuration when the time series are contaminated with $50 \%$ correlated observational noise. Moreover, note that for the maximal coupling strength considered in both Figs. 2 and 3 the oscillators are phase synchronized. If we increase further the coupling strength, the synchronization becomes almost complete, in which case it is not possible anymore to infer the correct coupling configuration.

Now we apply PARS analysis to the challenging case of a network of experimental data exhibiting multiple time scales. The data were obtained from chemical experiments with $\mathrm{Ni}$ electrodissolution in $4.5 \mathrm{~mol} / \mathrm{L}$ sulfuric acid. The experiments were carried out with three $1 \mathrm{~mm} \mathrm{Ni}$ wires (with a spacing of $2 \mathrm{~mm}$ ) embedded in epoxy so that the dissolution processes took place only at the ends. The potential of the electrodes was set with a potentiostat to $V=1.400 \mathrm{~V}$ vs a Hg$/ \mathrm{Hg}_{2} \mathrm{SO}_{4} /$ sat. $\mathrm{K}_{2} \mathrm{SO}_{4}$ reference elec-
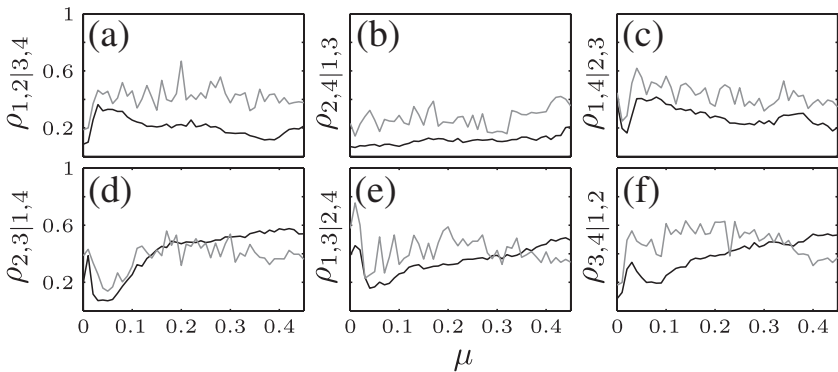

FIG. 3. Multivariate PARS analysis with significance levels (gray) of four interdependent funnel Rössler systems with coupling strengths $\mu_{1,2}=\mu_{2,4}=\mu_{1,4}=0$ and $\mu=\mu_{2,3}=\mu_{1,3}=$ $\mu_{3,4}>0$. All oscillators are contaminated by $50 \%$ of auto- and cross-correlated observational noise $\eta_{i}(t)=a \eta_{i}(t-1)+$ $\chi_{i}(t)+b \sum_{j \neq i} \chi_{j}(t)$ where $i=1, \ldots, 4, a=0.1, b=0.01$ and $\chi_{i}$ is standard Gaussian white noise. The PARS indices correctly determine both the indirect couplings (a)-(c) and the direct couplings (d)-(f). 


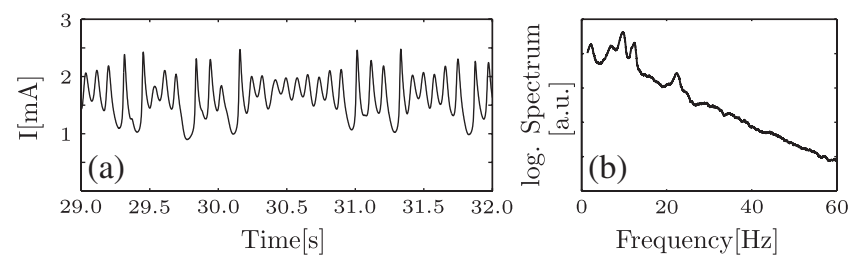

FIG. 4. (a) Segment of the time series and (b) power spectrum of current oscillations exhibiting multiple time scales of one of the three electrochemical oscillators.

trode in an electrochemical cell with a $1.57 \mathrm{~mm}$ diameter Pt coated Ti wire counter electrode. When $250 \Omega$ resistances were connected to each wire at $35^{\circ} \mathrm{C}$, the currents of the electrodes exhibited chaotic dynamics on multiple time scales as shown in Fig. 4, triggered by the hidden negative differential resistance caused by bisulfate ion adsorption $[13,14]$. The observed time series consisted of 38125 data points, sampled at $250 \mathrm{~Hz}$. The phase space was reconstructed by delay embedding with delay 20 and embedding 6 [11]. As discussed in [14], the electrodes did not show a significant level of synchrony without externally added interactions. Local interactions among the oscillators were introduced by a resistance $R$ inserted between electrodes 1 and 2 , and between electrodes 2 and 3, respectively. Note that a smaller resistance leads to a stronger coupling.

In Fig. 5 the results of the bivariate synchronization analysis and the multivariate PARS analysis are compared. The bivariate synchronization indices erroneously indicate interactions between all pairs of oscillators. But the multivariate PARS index $\rho_{1,3 \mid 2}$ does not become statistically significant, even for large coupling strengths [Fig. 5(e)], so that we can conclude that the connection between the oscillators $\{1,3\}$ is indirect, in agreement with the experimental setup.

In summary, partial recurrence based synchronization analysis has been introduced to generalize partial phase synchronization. PARS analysis allows distinguishing direct from indirect interactions in networks of oscillatory

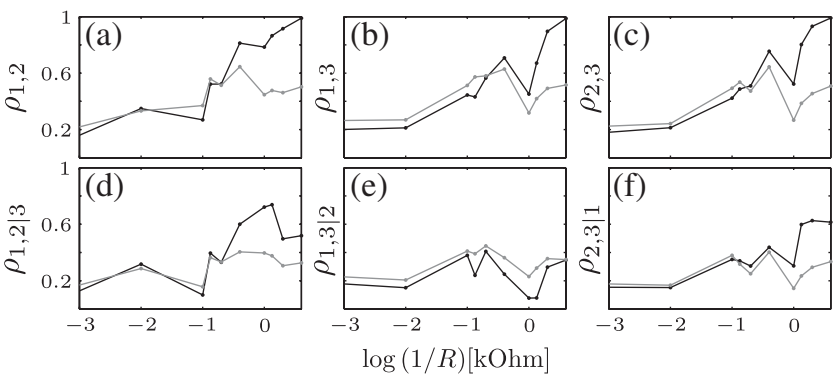

FIG. 5. (a)-(c) Bivariate analysis and (d)-(f) multivariate PARS analysis with significance levels (gray) of three electrochemical oscillators with varied local interactions depending on the resistance $R$. (e) PARS analysis correctly determines the indirect coupling between the oscillators $\{1,3\}$. systems with multiple time scales, where a straightforward definition of the phase is ambiguous or not possible. Here, we have focused on phase synchronization, but the methodology is applicable also to generalized synchronization and is very robust with respect to high levels of noise [5]. Moreover, we have validated the statistical significance of our results using twin surrogates. To illustrate the performance of the method, we have successfully applied it to model systems of oscillatory networks with multiple time scales and to experimental data from three non-phasecoherent electrochemical oscillators. The methodology proposed here will have a strong impact on complex networks research and its applications, in particular, to the detection of motifs embedded in larger networks.

This work was supported by the DFG (Ti315/4-2; J. K.: SFB 555, project C1) and by the Excellence Initiative of the German Federal and State Governments. B. S. is indebted to the Landesstiftung Baden-Württemberg for the financial support by the Eliteprogramme for Postdocs. M. C. R. would like to thank SULSA for financial support. M. T. was supported by the RCUK. I.Z.K. acknowledges support from Research Corporation.

*Corresponding author: jakob.nawrath@gmx.de

[1] O. Sporns and R. Kötter, PLoS Biol. 2, e369 (2004); M. Steriae, Trends Neurosci. 28, 317 (2005).

[2] J. Granger and M. Hatanaka, Spectral Analysis of Economic Time Series (Princeton University Press, Princeton, 1964).

[3] B. Schelter, M. Winterhalder, R. Dahlhaus, J. Kurths, and J. Timmer, Phys. Rev. Lett. 96, 208103 (2006).

[4] C. J. Stam and B.W. van Dijk, Physica (Amsterdam) 163D, 236 (2002).

[5] M. C. Romano, M. Thiel, J. Kurths, I.Z. Kiss, and J.L. Hudson, Europhys. Lett. 71, 466 (2005).

[6] This index can be extended to consider $n: m$ phase synchronization, see e.g., [8].

[7] F. Mormann, K. Lehnertz, P. David, and C.E. Elger, Physica (Amsterdam) 144D, 358 (2000).

[8] M. G. Rosenblum, A. S. Pikovsky, and J. Kurths, Phys. Rev. Lett. 76, 1804 (1996); A. Pikovsky, M. Rosenblum, and J. Kurths, Synchronization-A Universal Concept in Nonlinear Sciences (Cambridge University Press, Cambridge, 2001).

[9] J.-P. Eckmann, S. O. Kamphorst, and D. Ruelle, Europhys. Lett. 4, 973 (1987); N. Marwan, M. C. Romano, M. Thiel, and J. Kurths, Phys. Rep. 438, 237 (2007).

[10] G. V. Osipov, B. Hu, C. Zhou, M. V. Ivanchenko, and J. Kurths, Phys. Rev. Lett. 91, 024101 (2003).

[11] H. Kantz and T. Schreiber, Nonlinear Time Series Analysis (Cambridge University Press, Cambridge, England, 1997).

[12] M. Thiel, M. C. Romano, J. Kurths, M. Rolfs, and R. Kliegl, Europhys. Lett. 75, 535 (2006).

[13] M. T. M. Koper, Adv. Chem. Phys. 92, 161 (1996).

[14] I. Z. Kiss and J. L. Hudson, Phys. Chem. Chem. Phys. 4, 2638 (2002). 